\title{
Complaint Redressal Practices in Public and Private Insurance Companies
}

\author{
N.Nagalakshmi, S.P.Mathiraj
}

\begin{abstract}
The life insurance industry of India has 23 licenses -holders running their business in this sector. The Life Insurance Corporation of India (LICI), which is the only player in the public sector, the remaining area is covered by the 22 private sector companies. IRDAI has taken initiatives to provide effective grievance handling machinery to address the grievances of policyholders. Consumer dispute Redressal agency is efficient for handling complaints and easily accessible. This paper examines the regulations and guidelines framed by IRDAI for effective grievance handling and the study would provide some insights into the areas, specifically status of grievances in public and private life insurance companies (LIC, SBI, HDFC, Reliance Life and Bajaj Allianz) and the functioning of consumer dispute Redressal agencies of life insurance sectors.
\end{abstract}

Index terms: Grievance Cell, Policyholders and the Insurance Regulatory and Development Authority (IRDA). JEL Classifications: $\mathbf{G 2 2}$

\section{INTRODUCTION}

In an insurance industry, the Consumer Affairs Department of IRDA handles policyholders' (insurance consumers) grievances. The Grievance Cell looks into the complaints from policyholders against life insurance companies. A grievance without resolution leads to dissatisfaction beyond control. The Redressal of customer grievances is a pre - requisite for ensuing long term relationship with customers and customer loyalty in service industry like insurance. Life insurance contract is a long term contract between the life assured and insurance company. Both the parties are bound to fulfill obligations lies with them. An efficient insurer is one who has an effective mechanism not only for providing excellent service but for redressing the complaints of customers.

\section{REVIEW OF LITERATURE}

Jimin Hong \& S. Hun Seog (2019) we investigate the effects of life settlement, the securitization of life insurance contracts, on the primary insurance market. We analyze an economic model in which policyholders have different liquidity risks in an overlapping generation setting, and both policyholders and the monopolistic insurer incur liquidity costs. We find that, under some conditions, life settlement may lead to an increase in the insurance demand and a decrease in financing costs. The insurer will have a higher profit when the decrease in financing costs or the increase in demand is sufficiently large.

Revised Manuscript Received on September 14, 2019.

N.Nagalakshmi, Ph.D Research Scholar, Department of Corporate Secretaryship, Alagappa University, Karaikudi, Tamilnadu, India. (E-mail: lavanyaa2302@gmail.com)

Dr. S.P.Mathiraj, Associate Professor, Department of Corporate Secretaryship, Alagappa University, Karaikudi, Tamilnadu, India.

\section{STATEMENT OF THE PROBLEM}

The study focuses on the grievance Redressal Practices in Public and Private Insurance Companies. The Insurance Regulatory and Development Authority (IRDA) has been recognized to adjust the industry of both the life and non-life insurance in India. Grievances Redressal Cell and try to make certain immediate Redressal of the complaints received from the policyholders. Policyholders who have complaints against insurers are necessary to first approach of Grievance/Customer Complaints Cell of the concerned insurer (insurance companies). If they do not receive a reply of the company, they may approach the Grievance Cell of the IRDA.

\section{OBJECTIVES OF THE STUDY}

To examine the status of grievances of public and private life insurance companies.

To analyze the perception regarding the functioning of Consumer Dispute Redressal Agency among the policyholders.

\section{HYPOTHESES OF THE STUDY}

H1 There is no significant difference in factors influencing taking policy.

$\mathrm{H} 2$ There is no significant difference between Life insurance Companies and its Policies.

H3 There is no significant difference in Complaint Redressal Practices.

$\mathrm{H} 4$ There is no significant difference in Functions of Consumer Dispute Redressal Agency.

\section{METHOD OF DATA COLLECTION}

The present study is descriptive in nature and makes use of primary and secondary data. A structured questionnaire was drafted and given to 50 respondents in order to carry out the research. The secondary data was collected from annual reports of office of the governing Body of Insurance Council, IRDA, Books, journals and website information. 


\section{DETAILS ABOUT PRIMARY DATA}

Sample size

: $\quad 50$ Respondents

Area

Grievances/Procedure for Redressal mechanism/onsumer dispute redressal agency

$\begin{array}{lll}\text { Industry } & : & \text { Insurance } \\ \text { Respondents } & : & \text { Policyholders }\end{array}$

\section{TOOLS USED}

Primary Data: The collected data will be processed with the help of appropriate statistical tools. The researcher tentatively proposes to use descriptive analysis, Cross tabulation, t- test, Pearson correlation, Kendall's test.

Secondary Data: In order to analyze the data and draw conclusions on this study, various statistical tools like Mean, Standard Deviation and Co-efficient of variance have been used for analysis.

\section{PERIOD OF THE STUDY}

The study period for secondary data is five years starting from $2012-13$ to $2017-18$

\section{LIMITATIONS OF THE STUDY}

The study is confined only to Grievance Redressal of the Health (general) insurance customers in an Indian Health (General) insurance industry.

\section{STATUS OF GRIEVANCES OF PUBLIC AND PRIVATE LIFE INSURANCE COMPANIES}

The Grievances Cell of the Authority (IRDA) receives grievances from the policyholders against insurance companies. Such complaints are forwarded to the companies for resolution at their end. The insurance companies are required to keep the Grievances Cell of IRDA informed about the status of respective grievances and whether the complaints have been resolved or otherwise. Table 1 gives the perspective of the complaints received by the Authority.

Table-1

Status of Grievances in Life Insurance Companies

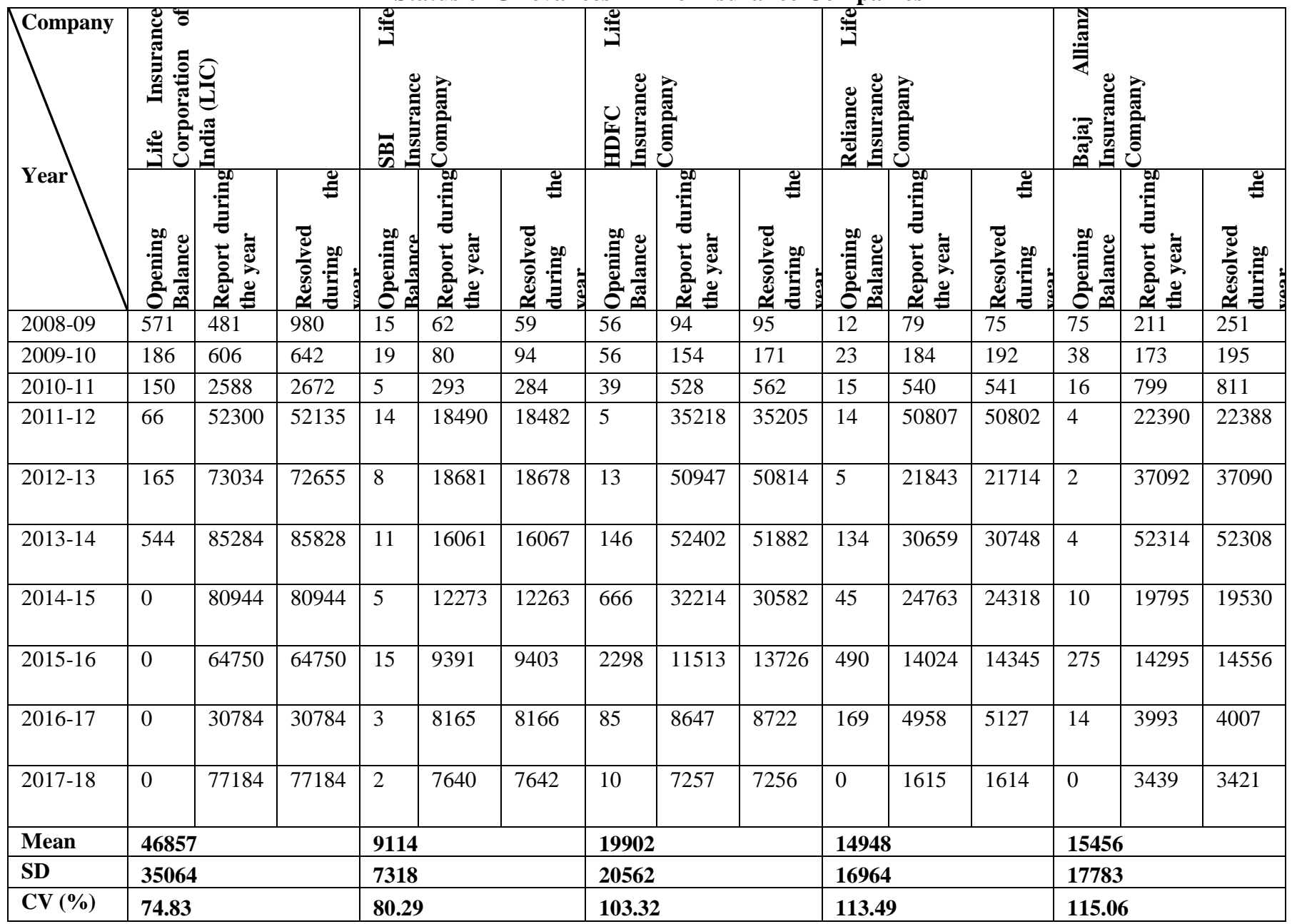

Source: IRDA Annual Report 
The Table 1 shows that status of grievance in selected life insurance companies. The Life Insurance Corporation India (LIC) mean value is 46857, standard deviation value is 35064 then the Co-variance Value is 74.83, SBI Life Insurance Company mean value is 9114 , standard deviation value is 7318 then the Co-variance Value is 80.29, HDFC Life Insurance Company mean value is 19902, standard deviation value is 20562 then the Co-variance Value is 103.32, Reliance Life Insurance company mean value is 14948, standard deviation value is 16964 then the Covariance Value is 113.49, Bajaj Allianz Life Insurance company mean value is 15456 , standard deviation value is 17783 then the Co-variance Value is 115.06.

\section{RESULTS AND DISCUSSION}

\section{Demographic Profile}

Socio economic profile is both quantitative and qualitative aspects of selected human population. The following section presents the social and economic profile of the respondents which includes Gender, Age, Marital Status, Occupation, Educational Qualification and Annual income.

Table- 2

Demographic Profile of the Respondents

\begin{tabular}{|c|c|c|c|}
\hline Social Factor & Classification & Frequency & Percentage $(\%)$ \\
\hline \multirow[t]{2}{*}{ Gender } & Male & 26 & 52.0 \\
\hline & Female & 24 & 48.0 \\
\hline \multirow[t]{5}{*}{ Age } & Below 30 Years & 5 & 10.0 \\
\hline & $31-40$ Years & 18 & 36.0 \\
\hline & 41-50 Years & 8 & 16.0 \\
\hline & 51-60 Years & 9 & 18.0 \\
\hline & Above 60 Years & 10 & 20.0 \\
\hline \multirow[t]{2}{*}{ Marital Status } & Unmarried & 20 & 40.0 \\
\hline & Married & 30 & 60.0 \\
\hline \multirow[t]{4}{*}{ Educational Qualification } & Illiterate & 4 & 8.0 \\
\hline & School Level & 16 & 32.0 \\
\hline & College Level & 21 & 42.0 \\
\hline & Professional & 9 & 18.0 \\
\hline \multirow[t]{6}{*}{ Occupational Level } & Government employee & 13 & 26.0 \\
\hline & Pvt. Sector employee & 10 & 20.0 \\
\hline & Self employed & 11 & 22.0 \\
\hline & Professional & 4 & 8.0 \\
\hline & Agriculturist & 8 & 16.0 \\
\hline & Retired Person & 4 & 8.0 \\
\hline \multirow[t]{3}{*}{ Income per annum } & Rs. $2,00,000$ to Rs. $4,00,000$ & 27 & 54.0 \\
\hline & Rs.4, 00,001 to Rs. $6,00,000$ & 18 & 36.0 \\
\hline & Above Rs.6, 00,000 & 5 & 10.0 \\
\hline
\end{tabular}

Source: Computed Data

The detailed respondent's demographic profile in terms of frequency \& percentage is presented in Table 2, 52\% respondents were male while $48 \%$ were female. Responded were mostly belong to age group 31-40 (36\%), followed by the age group Above 60 (20\%) \& 18\% respondent belong to age group 51-60. Mostly (60\%) of the respondents are married and $40(\%)$ respondents are Unmarried. Most of respondents $(26 \%, 13$ out of 50) are Government employee. $54 \%$ respondents have annual income in the range of 2 lakh- 4 lakhs.

Life insurance Companies and Policies 
Table- 3

Life Insurance Companies and its Policies

\begin{tabular}{|c|c|c|c|c|c|c|c|c|}
\hline \multicolumn{9}{|c|}{ Insurance Company $*$ Insurance policy Cross tabulation } \\
\hline & & & \multicolumn{5}{|c|}{ Insurance policy } & \multirow[b]{2}{*}{ Total } \\
\hline & & & \begin{tabular}{|l} 
Term \\
Policy
\end{tabular} & \begin{tabular}{|l} 
Money \\
Back \\
Policy \\
\end{tabular} & ULIP Plan & $\begin{array}{l}\text { Pension } \\
\text { Plan } \\
\end{array}$ & \begin{tabular}{|l} 
Chid \\
Benefit \\
Plan \\
\end{tabular} & \\
\hline \multirow{3}{*}{\multicolumn{2}{|c|}{ LIC }} & $\%$ within Insurance Company & $7.1 \%$ & & & $57.1 \%$ & $35.7 \%$ & $100.0 \%$ \\
\hline & & $\%$ within Insurance policy & $9.1 \%$ & & & $66.7 \%$ & $50.0 \%$ & $28.0 \%$ \\
\hline & & $\%$ of Total & $2.0 \%$ & & & $16.0 \%$ & $10.0 \%$ & $28.0 \%$ \\
\hline \multirow{3}{*}{\multicolumn{2}{|c|}{ SBI }} & $\%$ within Insurance Company & $80.0 \%$ & & & & $20.0 \%$ & $100.0 \%$ \\
\hline & & $\%$ within Insurance policy & $36.4 \%$ & & & & $10.0 \%$ & $10.0 \%$ \\
\hline & & $\%$ of Total & $8.0 \%$ & & & & $2.0 \%$ & $10.0 \%$ \\
\hline \multirow{9}{*}{ 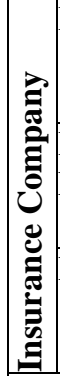 } & \multirow[t]{3}{*}{ Bajaj Allianz } & $\%$ within Insurance Company & $0.0 \%$ & $22.2 \%$ & $55.6 \%$ & $22.2 \%$ & & $100.0 \%$ \\
\hline & & $\%$ within Insurance policy & $0.0 \%$ & $33.3 \%$ & $45.5 \%$ & $16.7 \%$ & & $18.0 \%$ \\
\hline & & $\%$ of Total & $0.0 \%$ & $4.0 \%$ & $10.0 \%$ & $4.0 \%$ & & $18.0 \%$ \\
\hline & \multirow{3}{*}{$\begin{array}{l}\text { HDFC } \\
\text { Life }\end{array}$} & $1 . \%$ within Insurance Company & $42.9 \%$ & & $57.1 \%$ & & & $100.0 \%$ \\
\hline & & $\%$ within Insurance policy & $27.3 \%$ & & $36.4 \%$ & & & $14.0 \%$ \\
\hline & & $\%$ of Total & $6.0 \%$ & & $8.0 \%$ & & & $14.0 \%$ \\
\hline & \multirow[t]{3}{*}{ Reliance } & $\%$ within Insurance Company & $20.0 \%$ & $26.7 \%$ & $13.3 \%$ & $13.3 \%$ & $26.7 \%$ & $100.0 \%$ \\
\hline & & $\%$ within Insurance policy & $27.3 \%$ & $66.7 \%$ & $18.2 \%$ & $16.7 \%$ & $40.0 \%$ & $30.0 \%$ \\
\hline & & $\%$ of Total & $6.0 \%$ & $8.0 \%$ & $4.0 \%$ & $4.0 \%$ & $8.0 \%$ & $30.0 \%$ \\
\hline \multirow{3}{*}{\multicolumn{2}{|c|}{ Total }} & $\%$ within Insurance Company & $22.0 \%$ & $12.0 \%$ & $22.0 \%$ & $24.0 \%$ & $20.0 \%$ & $100.0 \%$ \\
\hline & & $\%$ within Insurance policy & $100.0 \%$ & $100.0 \%$ & $100.0 \%$ & $100.0 \%$ & $100.0 \%$ & $100.0 \%$ \\
\hline & & $\%$ of Total & $22.0 \%$ & $12.0 \%$ & $22.0 \%$ & $24.0 \%$ & $20.0 \%$ & $100.0 \%$ \\
\hline
\end{tabular}

Source: Computed Data

The Table 3 shows that $28.0 \%$ respondents have policies of LIC Company \& $10.0 \%$ respondents have policies of SBI Life, $18.0 \%$ respondents have policies of Bajaj Allianz, $14.0 \%$ respondents have policies of HDFC Std and 30.0\% respondents have policies of Reliance Life.

$22.0 \%$ respondents have Term policy out of which $27.3 \%$ plans are of Reliance Company. $66.7 \%$ respondents have Money Back policy, ULIP plan category, $18.2 \%$ and $16.7 \%$ \& $40 \%$ respondents have Pension Plans \& Child Benefits Plans.

Table- 3.1

Chi-Square Tests

\begin{tabular}{|c|c|c|c|}
\hline & Value & df & $\begin{array}{l}\text { Asymptotic } \\
\text { Significance } \\
\text { (2-sided) }\end{array}$ \\
\hline Pearson Chi-Square & $47.535^{\mathrm{a}}$ & 16 & .000 \\
\hline Likelihood Ratio & 54.577 & 16 & .000 \\
\hline $\begin{array}{l}\text { Linear-by-Linear } \\
\text { Association }\end{array}$ & 3.943 & 1 & .047 \\
\hline $\mathrm{N}$ of Valid Cases & 50 & & \\
\hline
\end{tabular}

\section{Source: Computed Data}

The calculated value of Chi-Square Tests is 47.535 and the $\mathrm{p}$ value is .000 . Since the calculated $\mathrm{P}$ value is higher than the 0.05 ( $\mathrm{P}$ value $>5 \%)$, the null hypothesis is accepted at the five percent level of significance. 
Factors Influencing taking the Insurance Policy

Table-4

Independent t- Test

\begin{tabular}{|c|c|c|c|c|c|c|c|c|}
\hline \multicolumn{9}{|c|}{ Independent t- Test } \\
\hline & & \multicolumn{2}{|c|}{\begin{tabular}{|c|} 
Levene's Test \\
for Equality of \\
Variances \\
\end{tabular}} & \multicolumn{5}{|c|}{ t-test for Equality of Means } \\
\hline & & $\mathbf{F}$ & Sig. & t & df & $P$ value & $\mathrm{MD}$ & $\begin{array}{c}\text { Std. Error } \\
\text { Diff }\end{array}$ \\
\hline \multirow[t]{2}{*}{ Premium } & Equal variances assumed & 7.670 & 0.008 & 2.132 & 48 & .038 & .288 & .135 \\
\hline & $\begin{array}{l}\text { Equal variances not } \\
\text { assumed }\end{array}$ & & & 2.145 & 47.843 & .037 & .288 & .135 \\
\hline \multirow[t]{2}{*}{ Charges } & Equal variances assumed & .980 & 0.327 & -.690 & 48 & .494 & -.112 & .163 \\
\hline & $\begin{array}{l}\text { Equal variances not } \\
\text { assumed }\end{array}$ & & & -.696 & 47.224 & .490 & -.112 & .161 \\
\hline \multirow[t]{2}{*}{ Policy Term } & Equal variances assumed & 1.300 & 0.260 & -.911 & 48 & .367 & -.199 & .218 \\
\hline & $\begin{array}{l}\text { Equal variances not } \\
\text { assumed }\end{array}$ & & & -.906 & 45.523 & .370 & -.199 & .219 \\
\hline \multirow[t]{2}{*}{ Savings } & Equal variances assumed & .960 & 0.332 & -.248 & 48 & .806 & -.038 & .155 \\
\hline & $\begin{array}{l}\text { Equal variances not } \\
\text { assumed }\end{array}$ & & & -.249 & 47.888 & .805 & -.038 & .155 \\
\hline \multirow[t]{2}{*}{ Accessibility } & Equal variances assumed & .032 & 0.860 & -4.275 & 48 & $.000^{*}$ & -.673 & .157 \\
\hline & $\begin{array}{l}\text { Equal variances not } \\
\text { assumed }\end{array}$ & & & -4.243 & 44.831 & .000 & -.673 & .159 \\
\hline \multirow[t]{2}{*}{ Company Image } & Equal variances assumed & 2.616 & 0.112 & .272 & 48 & .787 & .045 & .165 \\
\hline & $\begin{array}{l}\text { Equal variances not } \\
\text { assumed }\end{array}$ & & & .269 & 43.399 & .789 & .045 & .167 \\
\hline \multirow[t]{2}{*}{ Bonus and Interest } & Equal variances assumed & 12.667 & 0.001 & 1.732 & 48 & .090 & .218 & .126 \\
\hline & $\begin{array}{l}\text { Equal variances not } \\
\text { assumed }\end{array}$ & & & 1.750 & 46.500 & .087 & .218 & .125 \\
\hline \multirow{2}{*}{$\begin{array}{l}\text { Services(Pre \& Post } \\
\text { Sales) }\end{array}$} & Equal variances assumed & 4.054 & 0.050 & -1.671 & 48 & .101 & -.497 & .297 \\
\hline & $\begin{array}{l}\text { Equal variances not } \\
\text { assumed }\end{array}$ & & & -1.724 & 32.246 & .094 & -.497 & .288 \\
\hline \multirow[t]{2}{*}{ Wide risk coverage } & Equal variances assumed & 1.121 & 0.295 & .138 & 48 & .891 & .019 & .139 \\
\hline & $\begin{array}{l}\text { Equal variances not } \\
\text { assumed }\end{array}$ & & & .139 & 47.479 & .890 & .019 & .138 \\
\hline \multirow{2}{*}{$\begin{array}{l}\text { Quick settlement of } \\
\text { previous claims }\end{array}$} & Equal variances assumed & 1.084 & 0.303 & -1.151 & 46 & .256 & -.167 & .145 \\
\hline & $\begin{array}{l}\text { Equal variances not } \\
\text { assumed }\end{array}$ & & & -1.151 & 45.962 & .256 & -.167 & .145 \\
\hline
\end{tabular}

Source: Computed data

The Table 4 inferred that the Levene's test for equality of variances with a $\mathrm{p}$ value at $5 \%$ which is less than significance. It indicates the Factors influencing taking the insurance Policy are less than 5\%. Therefore, the null hypothesis is rejected.

Complaint Redressal Practices

Table-5

Complaint Redressal Practices

\begin{tabular}{|c|c|c|c|c|}
\hline \multicolumn{5}{|l|}{ Correlations } \\
\hline & & $\begin{array}{l}\text { Grievances } \\
\text { Redressal is } \\
\text { Major } \\
\text { Component }\end{array}$ & $\begin{array}{l}\text { Present } \\
\text { is grievance } \\
\text { handling } \\
\text { policy }\end{array}$ & $\begin{array}{l}\text { Complaint } \\
\text { handling/ } \\
\text { grievance } \\
\text { Redressal } \\
\text { prompt }\end{array}$ \\
\hline \multirow{3}{*}{$\begin{array}{l}\text { Grievances } \\
\text { Redressal is Major } \\
\text { Component }\end{array}$} & Pearson Correlation & 1 & & \\
\hline & Sig. (2-tailed) & & & \\
\hline & $\mathrm{N}$ & 50 & & \\
\hline \multirow{3}{*}{$\begin{array}{l}\text { Present grievance } \\
\text { handling policy }\end{array}$} & Pearson Correlation & .125 & 1 & \\
\hline & Sig. (2-tailed) & .388 & & \\
\hline & $\mathrm{N}$ & 50 & 50 & \\
\hline \multirow{3}{*}{\begin{tabular}{|ll} 
Complaint & handling/ \\
grievance & redressal \\
is prompt &
\end{tabular}} & Pearson Correlation & $.619^{* *}$ & .049 & 1 \\
\hline & Sig. (2-tailed) & .000 & .737 & \\
\hline & $\mathrm{N}$ & 50 & 50 & 50 \\
\hline
\end{tabular}

Source: Computed Data
According to Table - 5, the Complaint Redressal Practices has correlation with the variable such as Present grievance handling policy $(\mathrm{r}=.388, \mathrm{p}<0.01)$, Complaint handling/ grievance redressal is prompt $(\mathrm{r}=.000, \mathrm{p}<0.01)$.

Functioning of Consumer Dispute Redressal Agency

Table - 6

Functions of Consumer Dispute Redressal Agency

\begin{tabular}{|l|l|l|l|}
\hline S. & Functions & $\begin{array}{l}\text { Mea } \\
\mathbf{n} \\
\mathbf{R a n} \\
\mathbf{R}\end{array}$ & $\mathbf{R}$ \\
\hline 1 & $\begin{array}{l}\text { Consumer Dispute Redressal forums have economical } \\
\text { Redressal system }\end{array}$ & 8.27 & 11 \\
\hline 2 & $\begin{array}{l}\text { Consumer Dispute Redressal forums provide Speedy } \\
\text { trial mechanism }\end{array}$ & 9.45 & 2 \\
\hline 3 & $\begin{array}{l}\text { Consumer dispute Redressal forums are technically } \\
\text { efficient for handling Complaints }\end{array}$ & 8.80 \\
\hline 4 & $\begin{array}{l}\text { Consumer dispute Redressal decisions are regular and } \\
\text { consistent }\end{array}$ & 7.93 \\
\hline 5 & \begin{tabular}{l} 
Consumer dispute Redressal are easily accessible \\
\hline 6
\end{tabular} & $\begin{array}{l}\text { Consumer Dispute Redressal forums are fully transparent } \\
\mathbf{6 . 6 6}\end{array}$ & $\mathbf{1 6}$ \\
\hline 7 & $\begin{array}{l}\text { Consumer Dispute Redressal forums have impartial } \\
\text { judgment }\end{array}$ & 7.87 \\
\hline 8 & $\begin{array}{l}\text { Consumer Dispute Redressal forums have strong } \\
\text { execution of final order }\end{array}$ & 8.89 & 7 \\
\hline
\end{tabular}

execution of final order

Published By:

Blue Eyes Intelligence Engineering 


\begin{tabular}{|l|l|l|l|}
\hline 9 & $\begin{array}{l}\text { Consumer Dispute Redressal forums have systematic } \\
\text { mechanism }\end{array}$ & 8.88 & 8 \\
\hline 10 & $\begin{array}{l}\text { Consumer Dispute Redressal forums are compensatory in } \\
\text { nature }\end{array}$ & 6.76 & 15 \\
\hline 11 & Consumer Dispute Redressal forums have adequate staff & 7.45 & 14 \\
\hline 12 & $\begin{array}{l}\text { Consumer Dispute Redressal forums have hassle- } \\
\text { free(easy) dispute mechanism }\end{array}$ & 6.90 & 6 \\
\hline 13 & $\begin{array}{l}\text { Consumer Dispute Redressal forums have proper } \\
\text { infrastructure facility }\end{array}$ & 9.07 & 3 \\
\hline 14 & $\begin{array}{l}\text { Consumer Dispute Redressal forums settle cases within } \\
90 \text { days }\end{array}$ & 9.00 & 5 \\
\hline 15 & $\begin{array}{l}\text { Consumer Dispute Redressal forums strongly reject } \\
\text { frivolous and vexatious complaint }\end{array}$ & 8.73 & 10 \\
\hline 16 & $\begin{array}{l}\text { Consumer Dispute Redressal forums do consensus } \\
\text { judgments }\end{array}$ & 9.02 \\
\hline
\end{tabular}

Source: Computed Data

According to mean rank of functions of Consumer dispute Redressal Agency; the highest rank of this function is Consumer Dispute Redressal Agency is easily accessible and then the lowest rank is Consumer dispute Redressal agency is fully transparent.

Table - 6.1

Kendall's W Test

\begin{tabular}{|c|c|}
\hline \multicolumn{2}{|c|}{ Test Statistics } \\
\hline Kendall's W $^{\mathrm{a}}$ & .055 \\
\hline Chi-Square & 41.510 \\
\hline $\mathrm{df}$ & 15 \\
\hline Asymp. Sig. & .000 \\
\hline $\begin{array}{l}\text { a. Kendall's } \\
\text { Concordance }\end{array}$ & Coefficient of \\
\hline
\end{tabular}

Source: Computed Data

Kendall's W test has been administered to determine whether functions of Consumer Dispute Redressal agency have a different rank order preference for the different factor has influenced to Consumer Dispute Redressal agency. This functions $\mathrm{W}=.005$, which indicates there is no level of functions between the judges, and the $\mathrm{P}=0.000$ is less the $5 \%$ significant level, hence it is conclude the rejecting the null hypothesis that there is functions among the judges.

\section{CONCLUSION}

Complaint redress practices in very important role of the better customer relationship management. This study has been undertaken by status of grievances in selected life insurance companies, Life insurance Companies and Policies, Factors influencing taking the Insurance Policy, Complaint Redressal Practices and Functioning of Consumer Dispute Redressal Agency. Grievances of policyholders effectively and efficiently, insurance can create a situation of both life and non-life insurance companies.

\section{ACKNOWLEDGMENT}

One of the authors N.Nagalakshmi, gratefully acknowledges RUSA - Phase 2.0/Ph.D. Fellowship/2019. The corresponding author, Dr.SP.Mathiraj, gratefully acknowledge the RUSA - Phase 2.0 Grant for financial support to carry out this work.

\section{REFERENCES}

1 Shakeel-Ul-Rehman, D. I. (2012). Consumers' Grievance Redressal System in the Indian Life Insurance Industry An Analysis. South Asian Academic Research Journals, 2 (6), 215-227.

2 Seog, J. H. (2019). The efficiency effects of life settlement on the life insurance market. Pacific-Basin Finance Journal , 56, 395-412.

3 Prof. R. C. S. Rajpurohit \& Ranu Nawal (2016). Grievance Redressal Mechanism in Indian Life Insurance Industry: An Exploratory Study on Quantifying Relationships. Pacific Business Review International, 8 (7), 101-112.

4 IRDA Annual Report. 